\section{Häufiges Spucken - Hinweis auf Milchallergie}

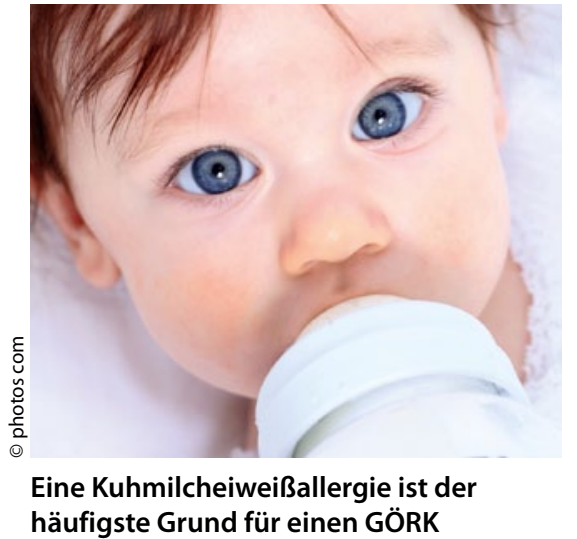

— Als Auslöser eines gastroösophagealen Refluxes (GÖRK) kommen Nahrungsallergien, Fehlbildungen, neurologische Erkrankungen oder eine zystische Fibrose in Betracht. Bei etwa der Hälfte der Kinder mit GÖRK ist die Ursache eine Kuhmilcheiweißallergie. Eine Kuhmilcheiweißallergie kann sich nicht nur als gastrointestinale Störung äußern, die dann selten lgE-vermittelt ist, berichtete $\mathrm{Dr}$. Kirsten Beyer von der Berliner Charité. Eine weitere potenzielle Manifestation ist die atopische Dermatitis. Möglich sind auch - meist lgE-assoziierte - Sofortallergien, die in vielen Organen Symptome auslösen können, etwa in Haut und Gewebe Juckreiz und QuinckeÖdem sowie im Atemtrakt Niesen oder Giemen. Da eine GÖRK am häufigsten auf einer Kuhmilchallergie basiert, empfahl Beyer deren Abklärung als erste diagnostische Maß- nahme. Für die Praxis schlug sie vor: Familienanamnese, Erheben der Symptome und eventuell Allergietests, die allerdings kein zuverlässiges Urteil erlauben.

Die beste Diagnosemethode sei daher eine Eliminationsdiät mit einer Nahrung aus freien non-allergenen Aminosäuren (Neocate ${ }^{\oplus}$ ), sagte Beyer. Haben sich die Beschwerden nach zwei bis vier Wochen gebessert, sei eine erneute sorgfältig überwachte Provokation mit Kuhmilch angezeigt. Bei eindeutigen oder schweren Sofortreaktionen und hohen lgE-Werten erfolgt für sechs bis 18 Monate eine therapeutische Diät mit der
Aminosäureformula. In Studien schrien die mit Aminosäureformula gefütterte Babys bereits nach zehn Tagen signifikant kürzer und spuckten seltener. Extensive Kuhmilchhydrolysate eignen sich Beyer zufolge nur bedingt für Diagnose und Behandlung der Kuhmilcheiweißallergie, weil sie eine Restallergenität besitzen, sodass $40 \%$ der Kinder weiter gastrointestinale Symptome haben. Schafs- oder Ziegenmilch sei wegen Kreuzreaktionen ebenfalls unangebracht. as

Symposium „Wenn Essen keinen Spaß mehr macht - Herausforderungen bei der Ernährung von Kindern"' anlässlich der 106. Jahrestagung der DGKJ. Potsdam, 17. September 2010. Veranstalter Nutricia, Erlangen

\section{Für ein gutes Bauchgefühl: Iberis-amara-Kombination}

Gastrointestinale Störungen wie funktionelle Dyspesie und Reizdarmsyndrom sind unter Kindern weit verbreitet. Mit dem Phytotherapeutikum Iberogast ${ }^{\oplus}$ ist eine Therapieoption verfügbar, die hervorragende und sehr umfangreiche Ergebnisse in der Behandlung von pädiatrischen Patienten mit funktionellen Magen-Darm-Erkrankungen vorweisen kann. Die Iberis-amara-Kombination wirkt multikausal und zeigt höchste Verträglichkeit.

Im Rahmen einer aktuellen prospektiven Studie wurde lberogast ${ }^{\oplus}$ zur Behandlung von Kindern im Alter von 3-14 Jahren mit funktionellen gastrointestinalen Störungen nach ROME III untersucht. Die behandelnden Ärzte ermittelten die Unter- und Oberbauchbeschwerden mithilfe eines gastrointestinalen Symptomscores (GIS). Die Studie belegte die hohe Wirksamkeit von Iberogast ${ }^{\oplus}$ bei pädiatrischen Patienten. Der Summenscore der Ober- und Unterbauchbeschwerden verringerte sich im Mittel bei allen Kindern von 16,1 \pm 8,93 Score-Punkten zu Beginn der Therapie auf 3,8 \pm 4,24 Punkte am Ende der Therapie. $86,6 \%$ der Ärzte gaben die Noten „sehr gut" bis „gut" für die Effektivität des Phytotherapeutikums.

Nach Informationen von Steigerwald, Darmstadt

\section{Babyhaut richtig pflegen}

Die Hautpflege von gesunden Säuglingen auf wissenschaftlich fundierte Füße zu stellen - daran wird an der Charité-Universitätsmedizin Berlin kontinuierlich gearbeitet. Vor kurzem hatte Prof. Dr. Ulrike Blume-Peytavi die ersten Pflegeempfehlungen vorgestellt. Sie waren unter Berücksichtigung der Ergebnisse einer europäischen Expertengruppe entwickelt worden:

_ Babys sollten zwei- bis dreimal pro Woche gebadet werden (in warmen Ländern auch öfter).

- Die Raumtemperatur sollte mind. $22^{\circ} \mathrm{C}$ betragen, die Wassertemperatur $37-38^{\circ} \mathrm{C}$. Das Bad sollte nicht länger als fünf bis zehn
Minuten dauern und das Kind anschließend sanft, aber rasch abgetrocknet werden, um eine Auskühlung zu vermeiden. _ Ein milder Badezusatz hat positive Auswirkungen auf die Babyhaut und reinigt besser als klares Wasser. Der Badezusatz sollte speziell für Babyhaut entwickelt sein, keine reizenden Substanzen enthalten und flüssig sein (keine alkalischen Seife).

_ Die Verwendung einer Babypflegecreme unterstützt und verbessert die Barrierefunktion der Haut.

Zusätzlich präsentierte Blume-Peytavi neue wissenschaftliche Untersuchungen: Erstens: Baden hat keinen negativen Einfluss auf die
Barrierefunktion der Haut. Außerdem führt es zu geringerem Wärmeverlust und größerem Wohlbefinden als Waschen. Zweitens: Milde Reinigungsprodukte beeinflussen den pH-Wert der Haut nicht negativ, alkalische Seifen dagegen erhöhen ihn, ein insbesondere im Säuglingsalter unerwünschter Effekt mit möglichen negativen Auswirkungen auf die Hautbarrierefunktion. Drittens: Die Haut ist bereits wenige Tage nach der Geburt natürlicherweise bakteriell besiedelt, was durch eine Anwendung von gängigen Waschregimen sowie Babyreinigungs- und Pflegemitteln nicht negativ beeinflusst wird.

Nach Informationen von Johnson \& Johnson, Norderstedt 